\title{
Analysis and Assessment on Distribution Voltage Sag Based on Stepped Current Protection
}

\author{
MA Li
}

School of Electrical Engineering, Xi' an University of Science \& Technology, Xi' an 710054,P.R.China

\begin{abstract}
The duration of voltage sag caused by fault is depended on the fault clearing time of the protection system. In traditional assessments on voltage sag are simply assumed that the duration obeys some distribution of random or is a constant value. In order to depict the duration of voltage sags more precisely, time characteristic of stepped current protection and protection scope of instantaneous current protection are taken into consideration, the two demarcation points of the protection operation on line are determined, the protection operation time of every segment is analyzed. An example is simulated by PSCAD/EMTDC software, and it is shown that more reasonable assessment is obtained by precise duration of voltage sag.

KEY WORDS: Voltage sag; duration; stepped current protection; protection zone; Assessment
\end{abstract}

\section{INTRODUCTION}

With the use of power electronic equipment increasing, electricity loads are more and more sensitive to voltage sag. Voltage sag has become one of the most important power quality problems in power system, Voltage sags caused by the user complaints account for the entire power quality problems complaints $80 \%$. Voltage sag will cause the program logic controller malfunction, speed regulating device disoperation, and computer data loss. Hence it is necessary to analyze and assess voltage sags reasonably.

The value and duration of voltage sag are two important characteristic quantities. In traditional assessments on voltage sag are simply assumed that the duration obeys some distribution of random or is a constant value[1], in traditional assessments on voltage sag are simply assumed that the duration obeys some distribution of random or is a constant value. Therefore it is necessary that the duration of voltage sag should be depicted precisely to obtain more reasonable and reliable assessment.

Generally the duration of voltage sag is depended on the fault clearing time of the protection system. The damage degree of voltage sag is influenced by the protection characteristic [2] and the fault clearing time of the protection operation. Stepped current protection[3] is used in distribution network[4], instantaneous current protection (I step) and time-limited instantaneous current protection (IIstep) are line main protection, over current protection (III step) is line backup protection. For this reason, the fault clearing time of line isn't simply assumed same, the duration of voltage sag should be depicted by time characteristic [5] of line protection.

In this paper, In order to depict the duration of voltage sags more precisely, time characteristic of stepped current protection and protection scope of instantaneous current protection are taken into consideration, two demarcation points of protection operation on line are determined, line is divided into three sections: I step protection operation section, IIstep protection operation section and the protection operation section to be determined. For I step and IIstep protection operation section, without considering the operation mode and fault type, the fault clearing time is determined quickly and accurately. The example simulated by PSCAD/EMTDC software demonstrates that more reasonable assessment is obtained by precise duration of voltage sag.

\section{TIME CHARACTERISTIC OF STEPPED CURRENT PROTECTION}

Because the whole line can't be protected by the instantaneous current protection, the end of line 1 is 
protected by time-limited instantaneous current protection, and the head of line 2 is protected by instantaneous current protection. The fault currents of the end of line 1 and the head of line 2 are almost the same, however, according to the time characteristic of stepped current protection, for the end of line 1 , the fault clearing time is more.

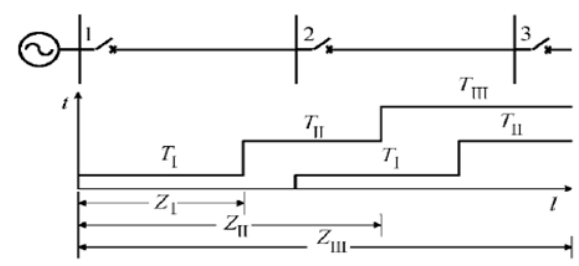

Figure1 Time characteristic of stepped current protection

\section{PROTECTION SCOPE OF INSTANTANEOUS CURRENT PROTECTION}

The duration of voltage sag caused by fault is depended on the fault clearing time of the protection system. When fault happen on line, the operation mode, system fault type and fault location should be taken into consideration to determine the fault clearing time.

Short circuit current is influenced by the operation mode, system fault type and fault location. Three phase short circuit current can be expressed as

$$
I_{k}=\frac{E_{\phi}}{Z_{s}+Z_{k}}
$$

Where $E_{\phi}$ is the phase voltage of equivalent source, $Z_{s}$ is the impedance between protection element mounting point and equivalent source, $Z_{k}$ is the impedance between fault point and protection element mounting point.

For the protection device, the operation mode under which short-circuit current of protection element mounting point is maximum is known as the maximum operation mode. The operation mode under which short-circuit current of protection element mounting point is minimum is known as the minimum operation mode. In order to ensure the selectivity of instantaneous current protection, starting current of the protection device must be larger than the current $I_{k \cdot \max }^{(3)}$ produced by the three-phase short circuit fault occurring on the end of protected line under the maximum operation mode.

$$
I_{k, \max }^{(3)}=\frac{E_{\phi}}{Z_{s, \text { max }}+z L}
$$

Where $Z_{s, \max }$ is the impedance between protection element mounting point and equivalent source under the maximum operation mode, $z$ is the unit impedance of line, $L$ is the length of line protected.

$$
I_{\text {set }}^{\mathrm{I}}=K_{r e l}^{\mathrm{I}} I_{k, \text { max }}^{(3)}
$$

Where $K_{r e l}^{\mathrm{I}}$ is the confidence coefficient, generally its value is $1.2 \sim 1.3$.

The maximum operation mode, the minimum operation mode and fault type are taken into consideration, protection zone of instantaneous current protection can be written as follows:

(1)Under the maximum operation mode three-phase short circuit occur, the protection zone is:

$$
l_{\max }^{(3)}=\frac{1}{z}\left(\frac{E_{\phi}}{I_{s e t}^{1}}-Z_{s, \max }\right)
$$

(2)Under the maximum operation mode two-phase short circuit occur, the protection zone is:

$$
l_{\max }^{(2)}=\frac{1}{z}\left(\frac{\sqrt{3} E_{\phi}}{2 I_{\text {set }}^{\mathrm{I}}}-Z_{s, \max }\right)
$$

(3)Under the minimum operation mode three-phase short circuit occur, the protection zone is:

$$
l_{\min }^{(3)}=\frac{1}{z}\left(\frac{E_{\phi}}{I_{s e t}^{1}}-Z_{s, \min }\right)
$$

Where $Z_{s, \text { min }}$ is the impedance between protection element mounting point and equivalent source under the minimum operation mode.

(4)Under the minimum operation mode two-phase short circuit occur, the protection zone is:

$$
l_{\min }^{(2)}=\frac{1}{z}\left(\frac{\sqrt{3} E_{\phi}}{2 I_{\text {set }}^{\mathrm{I}}}-Z_{s, \min }\right)
$$

\section{THE FAULT CLEARING TIME BASED ON STEPPED CURRENT PROTECTION}

Under the maximum operation mode, three-phase short circuit fault occurs, if the fault point is within the scope of $l_{\max }^{(3)}$ protection, the fault clearing time is the I step protection operation time, if the fault point is besides the scope of $l_{\max }^{(3)}$ protection, the fault clearing time is the II step protection operation time. Similarly, for each operation mode, the each fault type has the corresponding scope of protection in line.

Line $i j$ in Figure 2 as an example, under the maximum operation mode, three-phase short circuit fault occurs, the scope of $l_{\max }^{(3)}$ protection is maximum, its corresponding demarcation point is $N_{\text {imax }}$. Under the minimum operation mode, two-phase short circuit fault occurs, the scope of $l_{\min }^{(2)}$ 
protection is minimum, its corresponding demarcation point is $M_{i \min }$. Similarly, the corresponding demarcation point of $l_{\min }^{(3)}$ is $N_{i \min }$, the corresponding demarcation point of $l_{\max }^{(2)}$ is $M_{i \max }$.

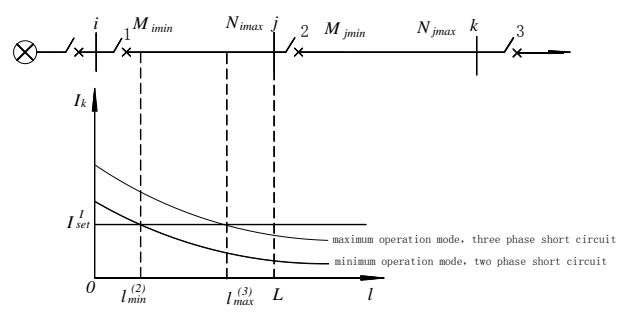

Figure 2 The protection scope and its corresponding demarcation point

From the Figure 2, line $i j$ is divided into three sections by $M_{i \min }$ and $N_{i \max }$. When the fault point is within $i M_{i \min }$ section, whether the system is what kind of operation mode, what type of short circuit, short circuit current is greater than the starting current $I_{\text {set }}^{\mathrm{I}}$, the fault clearing time is the I step protection operation time, for this reason, $i M_{i \min }$ section is known as the I step protection operation section. When the fault point is within $N_{i \max } j$ section, whether the system is what kind of operation mode, what type of short circuit, short circuit current is less than the starting current $I_{\text {set }}^{\mathrm{I}}$, the fault clearing time is the II step protection operation time, $N_{i \max } j$ section is the II step protection operation section. When the fault point is within $M_{i \min } N_{i \max }$ section, the operation mode, fault type and fault location should be taken into consideration to determine the fault clearing time, for this reason, $M_{i \min } N_{\text {max }}$ section is the protection operation section to be determined.

In the same way, line $\mathrm{jk}$ is divided into three sections by $M_{j \min }$ and $N_{j \max } . j M_{j \min }$ section is the I step protection operation section, $N_{j \max } k$ section is the II step protection operation section, $M_{\text {jmin }} N_{j \max }$ section is the protection operation section to be determined.

For two adjacent lines, if under same operation mode, the same type faults occur on $N_{i \max } j$ section and $j M_{j \min }$ section respectively, the values of voltage sag caused by the two faults are almost same, however, $N_{\text {max }} j$ section is II step protection operation section, $j M_{j \min }$ section is the I step protection operation section, the protection operation time of $N_{i \max } j$ section is longer, therefore, the voltage sag caused by the fault occurring on $N_{\text {imax }} j$ section is more serious. It can be seen that the voltage sag caused by the fault occurring on the end of line is more serious than that caused by fault occurring on the head of the adjacent next line.

\section{EXAMPLE ANALYSIS}

An example in this paper is shown in Figure 3, stepped current protection is used in $35 \mathrm{kV}$ line 16 and 67. I step protection operation time is $150 \mathrm{~ms}$, II step protection operation time is $550 \mathrm{~ms}$. Bus 5 is connected with a voltage sensitive loads PC, if the voltage is less than $60 \%$ and the duration is more than $240 \mathrm{~ms}$, PC will be affected to be loss of data.

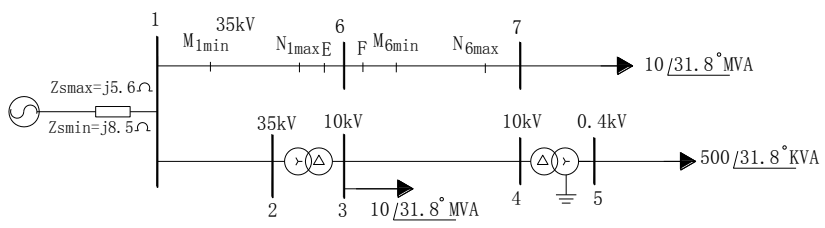

Figure 3 An example of voltage sag analysis based on stepped current protection

The analysis methods described above is used, the example is simulated by PSCAD/EMTDC software. When faults occur on line 16 and 67, the state of PC on bus 5 is analyzed and assessed. The scope of instantaneous current protection and protection operation section of line 16 and 67 are as shown in TABLE 1 and 2.

TABLE 1 The scope of instantaneous currnet protection of line 16 and $67(\mathrm{~km})$

\begin{tabular}{|c|c|c|c|c|}
\hline Line $i j$ & $l_{\max }^{(3)}=i N_{i \max }$ & $l_{\max }^{(2)}=i M_{i \max }$ & $l_{\min }^{(3)}=i N_{i \min }$ & $l_{\min }^{(2)}=i M_{i \min }$ \\
\hline Line 16 & 14.378 & 10.608 & 7.253 & 3.483 \\
\hline Line 67 & 23.548 & 15.87 & 16.423 & 8.745 \\
\hline
\end{tabular}

TABLE 2 The protection operation section of line 16 and $67(\mathrm{~km})$

\begin{tabular}{|c|c|c|c|}
\hline Line $i j$ & $i M_{i \min }$ & $N_{i \max } j$ & $M_{i \min } N_{i \max }$ \\
\hline Line 16 & 3.483 & 5.622 & 10.895 \\
\hline Line 67 & 8.745 & 1.452 & 14.803 \\
\hline
\end{tabular}

\subsection{For a line, if the fault clearing time is simply} assumed same, big error assessment results would be obtained.

(1) If the fault clearing time of line 16 is simply assumed $150 \mathrm{~ms}$, regardless of voltage sag value of bus 5 , the assessment results for the PC is without loss of data. In fact, when the fault point is within $N_{\text {Imax }} 6$ section, (II step protection operation section), that is the fault clearing time is $550 \mathrm{~ms}$, and $U_{5 s a g}<0.6$, the PC is loss of data. 
(2) If the fault clearing time of line 16 is simply assumed $550 \mathrm{~ms}$, when $U_{5 s a g}<0.6$, assessment results for the PC is loss of data, but in fact when the fault point is within $1 M_{\operatorname{lnin}}$ section (I step protection operation section), that is the fault clearing time is $150 \mathrm{~ms}$, the PC is without loss of data.

\subsection{The voltage sag caused by the fault occurring on the end of line is more serious than that caused by fault occurring on the head of the adjacent next line.}

Under the maximum operation mode, two three-phase short circuit faults occur on $E$ and $F$ respectively, the distances of $\mathrm{E}$ and $\mathrm{F}$ to bus 6 are $2 \mathrm{~km}$, as shown in Figure 3 . The voltage sags value of bus 5 caused by the two faults have little difference, they are less than 0.6 , but the PC state have nothing in common, as shown in TABLE 3. The voltage sag caused by the fault occurring on $\mathrm{E}$ is more serious than that caused by fault occurring on F. In this case, the main cause of PC of data loss is the fault clearing time.

TABLE 3 The state of pc on bus 5

\begin{tabular}{|c|c|c|c|}
\hline the fault point & $U_{\text {ssag }}$ & duration & PC state \\
\hline $\mathrm{E}$ & 0.53 & $550 \mathrm{~ms}$ & loss of data \\
\hline $\mathrm{F}$ & 0.57 & $150 \mathrm{~ms}$ & Without loss of data \\
\hline
\end{tabular}

5.3 For the protection operation section to be determined, the operation mode, fault type and fault location should be taken into consideration to determine the fault clearing time. When the fault point is within the corresponding protection scope, I step protection operate, $\mathrm{PC}$ is without loss of data, when the fault point is beside the corresponding protection scope, II step protection operate, if $U_{\text {sag }}<0.6, \mathrm{PC}$ is loss of data, if $U_{5 s a g}>0.6, \mathrm{PC}$ is without loss of data.

\section{CONCLUSION}

According to time characteristic of stepped current protection and protection scope of instantaneous current protection, two demarcation points of protection operation on line are determined, line is divided into three sections: I step protection operation section, II step protection operation section and the protection operation section to be determined. For I step and II step protection operation section, without considering the operation mode and fault type, the fault clearing time is determined quickly and accurately. The example demonstrates that more reasonable assessment is obtained by precise duration of voltage sag.

\section{REFERENCES}

[1] CHEN Li-pin, XIAO Xian-yong, ZHANG Zhi, et al. Voltage sags frequency assessment considering the time characteristic of protection system, Power System Protection and Control, 2013, 2012, VOL.41, (2): 113-118.

[2] CHEN Lipin, XIAO Xianyong, WANG Ying, et al. Assessment on Voltage Sag Frequency Considering Randomness of Protection Cooperation, Power System Technology, 2012, VOL.36, (5): 132-138.

[3] WANGBin, PANZhen-cunZ, XUWen-yuan, et al. Evaluations of Sag Mitigation Capability to Distribution Line Current Protection, Automation of Electric Power Systems, 2006, VOL.30(6): 25-29,41.

[4] Wang Bin, Panzhen-cun, Dongxin-zhou. A Scheme of the Fast Tripping Current Protection for Distribution Lines Based on the Voltage Sag Mitigation, Power System Technology, 2006, VOL.30 (21): 84-88.

[5] CHEN Lipin, XIAO Xianyong, WANG Ying, et al. Stochastic Evaluation of Voltage Sag Considering the Time Characteristic of Distance Protection in Meshed Network, Journal of Sichuan University (Engineering Science Edition), 2013, VOL.45 (3): 112-119. 